\title{
Association between Iodine Intake, Thyroid Function, and Papillary Thyroid Cancer: A Case-Control Study
}

\author{
Kyungsik Kim ${ }^{1,2,3}$, Sun Wook Cho ${ }^{4}$, Young Joo Park ${ }^{4,5}$, Kyu Eun Lee ${ }^{3,6,7,8}$, Dong-Wook Lee ${ }^{9}$, Sue K. Park ${ }^{1,3,8}$ \\ ${ }^{1}$ Department of Preventive Medicine, Seoul National University College of Medicine; ${ }^{2}$ Department of Biomedical Science, \\ Seoul National University Graduate School; ${ }^{3}$ Cancer Research Institute, Seoul National University; ${ }^{4}$ Department of Internal \\ Medicine, Seoul National University College of Medicine; ${ }^{5}$ Department of Molecular Medicine and Biopharmaceutical \\ Sciences, Graduate School of Convergence Science and Technology, Seoul National University; ${ }^{6}$ Department of Surgery, Seoul \\ National University Hospital, Seoul National University College of Medicine; ${ }^{7}$ Division of Surgery, Thyroid Center, Seoul \\ National University Cancer Hospital; ${ }^{8}$ Integrated Major in Innovative Medical Science, Seoul National University College of \\ Medicine, Seoul; ${ }^{9}$ Department of Family Medicine, Dongguk University College of Medicine, Gyeongju, Korea
}

Background: This study aimed to assess the effects of iodine intake, thyroid function, and their combined effect on the risk of papillary thyroid cancer (PTC) and papillary thyroid microcarcinoma (PTMC).

Methods: A case-control study was conducted including 500 community-based controls who had undergone a health check-up, and 446 overall PTC cases (209 PTC and 237 PTMC) from the Thyroid Cancer Longitudinal Study. Urinary iodine concentration (UIC), was used as an indicator of iodine intake, and serum for thyroid function. The risk of PTC and PTMC was estimated using unconditional logistic regression.

Results: Excessive iodine intake (UIC $\geq 220 \mu \mathrm{g} / \mathrm{gCr}$ ) was associated with both PTC (odds ratio [OR], $18.1395 \%$ confidence interval [CI], 8.87 to 37.04) and PTMC (OR, 8.02; 95\% CI, 4.64 to 13.87), compared to adequate iodine intake (UIC, 85 to $219 \mu \mathrm{g} / \mathrm{gCr}$ ). Free thyroxine (T4) levels $\geq 1.25 \mathrm{ng} / \mathrm{dL}$ were associated with PTC (OR, 1.97; 95\% CI, 1.36 to 2.87) and PTMC (OR, 2.98; 95\% CI, 2.01 to 4.41), compared to free T4 levels of 0.7 to $1.24 \mathrm{ng} / \mathrm{dL}$. Individuals with excessive iodine intake and high free T4 levels had a greatly increased OR of PTC (OR, 43.48; 95\% CI, 12.63 to 149.62), and PTMC (OR, 26.96; 95\% CI, 10.26 to 70.89), compared to individuals with adequate iodine intake and low free T4 levels.

Conclusion: Excessive iodine intake using creatinine-adjusted UIC and high free T4 levels may have a synergistic effect on PTC and PTMC. Considering both iodine intake and thyroid function is important to assess PTC and PTMC risk.

Keywords: Thyroid cancer, papillary; Papillary thyroid microcarcinoma; Iodine; Thyroid function tests

\section{INTRODUCTION}

Compared with other countries, the incidence rate and prevalence of thyroid cancer are relatively high in South Korea [1]. These high rates are thought to be due to a combination of detection bias, genetic factors, and environmental factors [2,3].
Risk factors include $\mathrm{X}$-radiation, gamma rays, radioactive iodine (I-131), family history of thyroid cancer, and benign thyroid disease have been reported [3].

Dietary iodine intake has also been suggested as a risk factor for thyroid cancer [3]. Iodine is an essential micronutrient and a vital component of the thyroid hormones, thyroxine (T4), and
Received: 13 March 2021, Revised: 28 June 2021, Accepted: 2 July 2021

Corresponding author: Sue K. Park

Department of Preventive Medicine, Seoul National University College of Medicine, 103 Daehak-ro, Jongno-gu, Seoul 03080, Korea

Tel: +82-2-740-8338, Fax: +82-2-747-4830, E-mail: suepark@snu.ac.kr
Copyright $\odot 2021$ Korean Endocrine Society

This is an Open Access article distributed under the terms of the Creative Commons Attribution Non-Commercial License (https://creativecommons.org/ licenses/by-nc/4.0/) which permits unrestricted non-commercial use, distribution, and reproduction in any medium, provided the original work is properly cited. 
triiodothyronine (T3), that are involved in various biochemical and metabolic pathways throughout the human body [4]. Thyroid hormones maintain homeostasis through the thyroid function feedback loop between the hypothalamus-pituitary axis and the thyroid gland. Even if there is a temporary excess or insufficient intake of iodine, thyroid hormones are in a balanced state. However, both chronic iodine deficiency and excess can lead to thyroid dysfunction by interfering with homeostasis [4-6].

Traditionally, thyroid cancer has been reported in areas where iodine intake was deficient. After iodine intake increased due to the salt iodization and iodine supplementation, the incidence of thyroid cancer, especially papillary thyroid cancer (PTC) increased despite of decrease in the incidence of follicular thyroid cancer [6-8]. In contrast, in some meta-analyses of the association between iodine intake and thyroid cancer have found that higher iodine intake is associated with a lower risk of thyroid cancer [9-11]. Therefore, there is controversy about the relationship between iodine intake and thyroid cancer. The inconsistencies between previous studies could be due to information bias and measurement error in dietary questionnaires, and inaccuracies in data on dietary iodine intake, differences in dietary sources of iodine by ethnicity and country, and differences in the characteristics of the study participants. In addition, considering the complicated link between recent and chronic dietary iodine intake and thyroid function, the inconsistencies between studies could be due to a complex interaction between factors which modify the risk of thyroid cancer [12-14]. However, it is unclear whether a high iodine intake directly induces PTC development, or indirectly contributes to PTC risk through interaction with thyroid hormones.

Therefore, the objective of this study was to evaluate the effects of iodine intake, measured using urinary iodine concentration (UIC), and thyroid function included thyroid stimulating hormone (TSH), free T4 and T3 on the risk of PTC and papillary thyroid microcarcinoma (PTMC). A second objective was to assess whether there was combined effect between UIC and thyroid hormones in determining the risk of PTC and PTMC.

\section{METHODS}

\section{Study design and population}

This study was a case-control study, and cases were participants in the Thyroid Cancer Longitudinal Study, a prospective study which has been reported in detail previously [15]. We primarily included 3,184 cases with PTC or PTC with follicular variant on histology record. We excluded participants who did not have both blood and urine samples ( $n=987)$; with insufficient information in potential confounders $(n=1,565)$; or those for whom were not matched with controls $(n=132)$. Of remained 500 PTC cases, we additionally excluded 54 cases due to hemolysis of the blood samples, or tumor size $<5 \mathrm{~mm}$. After these exclusions, there were a total of 446 PTC cases, including 209 participants with PTC (tumor size $\geq 10 \mathrm{~mm}$ ) and 237 participants with PTMC (tumor size 5 to $<10 \mathrm{~mm}$ ) (Supplemental Fig. S1).

The controls were individuals aged 20 to 80 years living in four urban regions who undergone a health check-up at regional medical institutions, public health centers, or temporary health check-up sites (e.g., town halls, community service centers). Regardless of the type of center, health check-up protocols were the same. Of 13,688 participants, we excluded those who did not have both blood and urine samples $(n=7,810)$; with insufficient information in confounders $(n=3,473)$; those who had previous cancer history or had taken medication that affects thyroid function for $\geq 3$ months $(n=1,598)$; or participants who were not matched with cases $(n=307)$. Finally, 500 controls were selected (Supplemental Fig. S1). Informed consent was waived by the Institutional Review Board of Seoul National University Hospital (IRB Number: 1603-102-750).

\section{Data collection}

Cases were enrolled during the preoperative period which in advance to surgery or other treatments, and controls were enrolled at the visit to health check-up center. Information such as demographic variables, dietary intake and other risk factors were collected by trained interviewers using structured questionnaires on enrollment. Blood and urine samples were collected after an 8 -hour fast and stored at $-70^{\circ} \mathrm{C}$ deep freezers in $1 \mathrm{~mL}$ aliquots. We measured iodine levels (unit: $\mu \mathrm{g} / \mathrm{L}$ ) using frozen $1 \mathrm{~mL}$ urine by inductively coupled plasma mass spectrometry (ICP-MS, 7900x, Agilent, Santa Clara, CA, USA); urinary creatinine was measured using Jaffe (C702, Roche, Mannheim, Germany); and serum sodium and potassium were measured using indirect ion selective electrode (ISE, Roche). Serum TSH, free T4, and T3 were measured using electrochemiluminescence immunoassays (E801, Roche).

\section{Stability of urine and serum samples for UIC and thyroid function}

In order to check the UIC stability in stored urine samples, UIC was measured in the urine of nine participants after storage for $1,7,30,90$, and 180 days. Repeated measures analysis of variance confirmed that there was no difference in UIC level ac- 
cording to the storage duration $(P=0.82)$. Preoperative stored samples from nine PTC cases, their results of the thyroid function were identified in the patients' medical records. The reliability between thyroid hormone levels identified in medical record and those newly-analyzed in stored frozen serum was excellent (intra-class correlation coefficients: 0.935, 0.876, and 0.812 for free T4, T3, and TSH, respectively).

\section{Statistical analysis}

The creatinine-adjusted and unadjusted UICs were classified into three and four groups, respectively: creatinine-adjusted $\mathrm{UIC},<85 \mu \mathrm{g} / \mathrm{gCr}$ (deficiency), 85-219 $\mu \mathrm{g} / \mathrm{gCr}$ (adequate), and $\geq 220 \mu \mathrm{g} / \mathrm{gCr}$ (excessive) [16]; unadjusted UIC, $<100 \mu \mathrm{g} / \mathrm{L}$ (deficiency), 100-199 $\mu \mathrm{g} / \mathrm{L}$ (adequate), 200-299 $\mu \mathrm{g} / \mathrm{L}$ (above requirements), and $\geq 300 \mu \mathrm{g} / \mathrm{L}$ (excessive) [17]. The UIC of $300 \mu \mathrm{g} / \mathrm{L}$ corresponds to iodine intake of $450 \mu \mathrm{g} / \mathrm{day}$, which corresponds to six cups of plain yogurt, eight cups of milk, and 20 slices of white bread, [13,18,19]. The thyroid function levels of each type of thyroid function indicators were classified into 'low,' 'normal,' and 'high' groups according to 'Seoul National University Hospital Clinical Lab' reference range as clinical cutpoints [20] and two groups according to population cutpoints [21]. The population cutpoints were the mean value of each hormone (TSH, $2.16 \mu \mathrm{IU} / \mathrm{mL}$; free T4, $1.25 \mathrm{ng} / \mathrm{dL}$ ), derived from data the Korea National Health and Nutrition Examination Survey, 2013 to 2015 (KNHANES) [21]. Because T3 information was not available from KNHANES, the population cutpoints for T3 were derived from the controls in this study.

The risk of overall PTC including each PTC and PTMC were estimated using multivariable logistic regression. Potential confounders were selected based on being statistically significant in univariate logistic regression with overall PTC or each main explanatory variable (UIC, free T4, T3, and TSH). Additionally, to assess the relationship between PTC and iodine intake, thyroid function graphically, we conducted spline analysis as visualization. We conducted stratified analyses according to sex, body mass index (BMI), and menopausal status among women. Caseonly analyses were conducted among overall PTC to assess the association between clinicopathological aggressiveness and UIC and thyroid hormone levels. Three measures of clinicopathological aggressiveness were used: the presence of the BRAF mutation, lymph node metastasis, and extra-thyroidal extension. To assess the combined effect of iodine intake and thyroid function, we made the four combination considering 'adequate' and 'excessive' creatinine-adjusted UICs and 'low' and 'high' free T4 or TSH using population cutpoints. To evalu- ate the performance on PTC discrimination according to explanatory variables, we calculated the area under the receiver operating characteristic curve (AUC) for three different types of models. Additionally, we assessed the statistical difference between models. Three models were constructed as follows:

Model 1: Function $(\mathrm{Y})=\beta_{0}+\beta_{1}$ (UIC)

Model 2: Function $(\mathrm{Y})=\beta_{0}+\beta_{1}(\mathrm{UIC})+\beta_{2}($ free T4 $)+\beta_{3}(\mathrm{TSH})$

Model 3: Function $(Y)=\beta_{0}+\beta_{1}(\mathrm{UIC})+\beta_{2}\left(\right.$ free T4) $+\beta_{3}$

$(\mathrm{TSH})+\beta_{4}$ (age) $+\beta_{5}($ sex $)+\beta_{6}($ total energy intake $)+\beta_{7}$ (education) $+\beta_{8}$ (family history of cancer) $+\beta_{9}$ (benign thyroid disease $)+\beta_{10}$ (dyslipidemia),

Function $(\mathrm{Y})=\operatorname{Ln}\left(\frac{P_{i}}{1-P_{i}}\right)$

All statistical analyses were performed using SAS version 9.4 (SAS Institute, Cary, NC, USA) or R software version 4.0 (RStudio, R Foundation for Statistical Computing, Vienna, Austria).

\section{RESULTS}

The characteristics of the cases of PTC and PTMC and the controls are shown in Table 1. Compared to the controls, patients with PTC or PTMC had higher education levels (both $P<$ 0.001 ), and a higher prevalence of family history of cancer in PTMC only $(P=0.003)$, dyslipidemia (both $P<0.001)$, and benign thyroid disease $(P=0.01, P<0.001)$. The mean BMI $(P=0.01)$ and the proportion of women who had ever been pregnant were lower in PTC patients than in the control group $(P=0.002)$, and the proportion of postmenopausal women was lower in PTMC patients than in the control group $(P=0.02)$. The distribution of two matching variables (age and sex) were not different between the PTC cases and the controls or between the PTMC cases and the controls.

Table 2 shows the association between case status and UIC. There was a higher prevalence of excessive iodine intake (UIC $\geq 220 \mu \mathrm{g} / \mathrm{gCr}$; UIC $\geq 300 \mu \mathrm{g} / \mathrm{L})$ in PTC cases and PTMC cases than controls. In addition, insufficient iodine intake (UIC $<85$ $\mu \mathrm{g} / \mathrm{gCr}$; UIC <100 $\mu \mathrm{g} / \mathrm{L}$ ) was rare in both the PTC cases and PTMC cases, but little bit higher in the control group. Excessive UIC ( $\geq 220 \mu \mathrm{g} / \mathrm{gCr}$ ) were associated with high risk of PTMC and PTC (odds ratio [OR], 8.02; 95\% confidence interval [CI], 4.64 to 13.87 ; and OR, 18.13 ; 95\% CI, 8.87 to 37.04 , respectively), and the strength of the association was greater for PTC than for PTMC ( $P$-ordinal <0.05). An excessive unadjusted UIC was also associated with an increased risk of PTC and PMTC analyses according to sex, BMI levels and women's menopausal sta- 
Table 1. Selected Characteristics between Controls and PTC Cases or Controls and PTMC Cases

\begin{tabular}{|c|c|c|c|c|c|}
\hline Characteristic & Controls $(n=500)$ & PTC $(n=209)$ & $P$ value ${ }^{a}$ & $\mathrm{PTMC}^{\mathrm{b}}(n=237)$ & $P$ value \\
\hline Age, yr & $47.8 \pm 11.3$ & $47.7 \pm 12.4$ & 0.92 & $47.6 \pm 11.6$ & 0.79 \\
\hline Body mass index, $\mathrm{kg} / \mathrm{m}^{2}$ & $24.0 \pm 3.1$ & $23.3 \pm 3.4$ & 0.01 & $23.6 \pm 3.3$ & 0.11 \\
\hline Female sex & $376(75.2)$ & $153(73.2)$ & 0.58 & $187(78.9)$ & 0.27 \\
\hline Education ( $\geq$ college) & $102(20.4)$ & $111(53.1)$ & $<0.001$ & $140(59.1)$ & $<0.001$ \\
\hline Ever cigarette smokers & $107(21.4)$ & $44(21.1)$ & 0.91 & $36(15.2)$ & 0.051 \\
\hline Ever alcohol drinkers & $258(51.6)$ & $101(48.3)$ & 0.43 & $131(55.3)$ & 0.35 \\
\hline Regular exercised & $203(40.6)$ & $91(43.5)$ & 0.47 & $90(38.1)$ & 0.52 \\
\hline Family history of cancer & $125(25.0)$ & $66(31.6)$ & 0.07 & $84(35.6)$ & 0.003 \\
\hline \multicolumn{6}{|l|}{ Past history of } \\
\hline Hypertension & $82(16.4)$ & $53(25.4)$ & 0.01 & $34(14.4)$ & 0.47 \\
\hline Dyslipidemia & $19(3.8)$ & $35(16.8)$ & $<0.001$ & $31(13.1)$ & $<0.001$ \\
\hline Benign thyroid disease & $14(2.8)$ & $15(7.2)$ & 0.01 & $23(9.7)$ & $<0.001$ \\
\hline Diabetes & $38(7.6)$ & $13(6.2)$ & 0.52 & $15(6.3)$ & 0.53 \\
\hline \multicolumn{6}{|l|}{ Females only } \\
\hline Pregnancy & $342(91.0)$ & $120(79.5)$ & 0.002 & $166(89.3)$ & 0.52 \\
\hline Post-menopausal & $184(48.9)$ & $73(47.7)$ & 0.80 & $72(38.5)$ & 0.02 \\
\hline
\end{tabular}

Values are expressed as mean \pm standard deviation or number (\%).

PTC, papillary thyroid cancer; PTMC, papillary thyroid microcarcinoma.

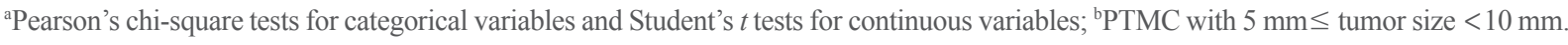

Table 2. Urinary Iodine Concentration for the Risk of PTC and PTMC

\begin{tabular}{|c|c|c|c|c|c|c|}
\hline \multicolumn{2}{|l|}{ Variable } & Controls $(n=500)$ & $\operatorname{PTC}(n=209)$ & OR $(95 \% \mathrm{CI})^{\mathrm{a}}$ & $\operatorname{PTMC}^{\mathrm{b}}(n=237)$ & OR $(95 \% \mathrm{CI})^{\mathrm{a}}$ \\
\hline \multicolumn{7}{|c|}{$\mathrm{UIC}, \mu \mathrm{g} / \mathrm{gCr}$} \\
\hline$<85$ & Insufficient iodine intake & $37(7.4)$ & 0 & $0.25(0.01-4.42)^{\mathrm{c}}$ & $1(2.5)$ & $0.17(0.02-1.45)$ \\
\hline $85-219$ & Adequate & $199(39.8)$ & $10(4.8)$ & 1.00 & $23(9.7)$ & 1.00 \\
\hline$\geq 220$ & Excessive & $264(52.8)$ & $199(95.2)$ & $18.13(8.87-37.04)$ & $213(87.8)$ & $8.02(4.64-13.87)$ \\
\hline \multicolumn{7}{|l|}{$\mathrm{UIC}, \mu \mathrm{g} / \mathrm{L}$} \\
\hline $20-99$ & Insufficient & $55(11.0)$ & $3(1.4)$ & $1.10(0.25-4.76)$ & $2(0.8)$ & $0.36(0.07-1.76)$ \\
\hline $100-199$ & Adequate & $146(29.2)$ & $6(2.9)$ & 1.00 & $13(5.5)$ & 1.00 \\
\hline $200-299$ & Above requirements & $82(16.4)$ & $5(2.4)$ & $1.09(0.31-3.89)$ & $12(5.1)$ & $1.57(0.62-3.96)$ \\
\hline$\geq 300$ & Excessive & $217(43.4)$ & $195(93.3)$ & $20.16(8.49-47.88)$ & $210(88.6)$ & $10.51(5.46-20.23)$ \\
\hline \multicolumn{7}{|l|}{$\mathrm{UIC}, \mu \mathrm{g} / \mathrm{L}$} \\
\hline $100-299$ & Adequate or above requirements & $228(51.2)$ & $11(5.3)$ & 1.00 & $25(10.6)$ & 1.00 \\
\hline$\geq 300$ & Excessive & $217(48.8)$ & $195(94.7)$ & $19.45(9.92-38.13)$ & $210(89.4)$ & $8.76(5.23-14.66)$ \\
\hline
\end{tabular}

Values are expressed as number $(\%)$.

PTC, papillary thyroid cancer; PTMC, papillary thyroid microcarcinoma; OR, odds ratio; CI, confidence interval; UIC, urinary iodine concentration. ${ }^{a}$ Adjusted for age, sex, education level, family history of cancer, past history of chronic disease (dyslipidemia and benign thyroid disease), and total energy intake; ${ }^{\text {bPTMC }}$ with $5 \mathrm{~mm} \leq$ tumor size $<10 \mathrm{~mm}$; ${ }^{\circ}$ Logit estimation in Cochran-Mantel-Haenszel method.

tus (Supplemental Table S1). In spline analysis, a positive association between UIC and PTC risk was observed, supporting the results of excessive UIC (Supplemental Fig. S2A).
Relative to normal levels of free T4, high free T4 levels were associated with a higher risk of PTMC and PTC (population cutpoints: OR, 2.98; 95\% CI, 2.01 to 4.41 ; and OR, 1.97; 95\% 
Table 3. Thyroid Function for the Risk of PTC and PTMC

\begin{tabular}{|c|c|c|c|c|c|}
\hline Variable & Controls $(n=500)$ & PTC $(n=209)$ & OR $(95 \% C I)^{a}$ & $\operatorname{PTMC}^{\mathrm{b}}(n=237)$ & OR $(95 \% \mathrm{CI})^{\mathrm{a}}$ \\
\hline \multicolumn{6}{|l|}{$\mathrm{TSH}, \mu \mathrm{IU} / \mathrm{mL}$} \\
\hline \multicolumn{6}{|c|}{ Clinical cutpoint ${ }^{\mathrm{c}}$} \\
\hline$<0.4$ & $14(2.8)$ & $7(3.4)$ & $1.60(0.59-4.34)$ & $10(4.2)$ & $0.92(0.33-2.60)$ \\
\hline $0.4-4.1$ & $457(91.4)$ & $180(86.1)$ & 1.00 & $209(88.2)$ & 1.00 \\
\hline$\geq 4.2$ & $29(5.8)$ & $22(10.5)$ & $1.64(0.85-3.19)$ & $18(7.6)$ & $1.08(0.53-2.21)$ \\
\hline \multicolumn{6}{|c|}{ Population cutpoint $^{\mathfrak{c}}$} \\
\hline $0.4-2.15$ & $337(69.3)$ & $123(60.9)$ & 1.00 & $136(59.9)$ & 1.00 \\
\hline$\geq 2.16$ & $149(30.7)$ & $79(39.1)$ & $1.37(0.93-2.01)$ & $91(40.1)$ & $1.39(0.95-2.05)$ \\
\hline \multicolumn{6}{|l|}{ Free T4, ng/dL } \\
\hline \multicolumn{6}{|c|}{ Clinical cutpoint ${ }^{\mathrm{c}}$} \\
\hline$<0.70$ & $2(0.4)$ & $1(0.5)$ & $2.06(0.18-23.37)$ & $1(0.4)$ & $1.61(0.14-18.54)$ \\
\hline $0.70-1.80$ & $494(98.8)$ & $189(90.4)$ & 1.00 & $216(91.2)$ & 1.00 \\
\hline$\geq 1.81$ & $4(0.8)$ & $19(9.1)$ & $12.79(3.98-41.18)$ & $20(8.4)$ & $12.46(3.71-41.88)$ \\
\hline \multicolumn{6}{|c|}{ Population cutpoint ${ }^{\mathrm{c}}$} \\
\hline $0.7-1.24$ & $265(53.2)$ & $74(35.6)$ & 1.00 & $68(28.8)$ & 1.00 \\
\hline$\geq 1.25$ & $233(46.8)$ & $134(64.4)$ & $1.97(1.36-2.87)$ & $168(71.2)$ & $2.98(2.01-4.41)$ \\
\hline \multicolumn{6}{|l|}{$\mathrm{T} 3, \mathrm{ng} / \mathrm{mL}$} \\
\hline \multicolumn{6}{|c|}{ Clinical cutpoint ${ }^{\mathrm{c}}$} \\
\hline$<0.87$ & $17(3.4)$ & $10(4.8)$ & $0.85(0.34-2.14)$ & $10(4.2)$ & $1.00(0.38-2.60)$ \\
\hline $0.87-1.84$ & $479(95.8)$ & $197(94.3)$ & 1.00 & $224(94.5)$ & 1.00 \\
\hline$\geq 1.85$ & $4(0.8)$ & $2(0.9)$ & $1.08(0.17-6.74)$ & $3(1.3)$ & $0.85(0.15-4.82)$ \\
\hline \multicolumn{6}{|c|}{ Population cutpoint ${ }^{d}$} \\
\hline $0.87-1.19$ & $214(44.3)$ & $105(52.8)$ & 1.00 & $114(50.2)$ & 1.00 \\
\hline$\geq 1.20$ & $269(55.7)$ & $94(47.2)$ & $0.79(0.55-1.14)$ & $113(49.8)$ & $0.91(0.63-1.31)$ \\
\hline
\end{tabular}

Values are expressed as number $(\%)$.

PTC, papillary thyroid cancer; PTMC, papillary thyroid microcarcinoma; OR, odds ratio; CI, confidence interval; TSH, thyroid stimulating hormone; T4, thyroxine; T3, triiodothyronine.

aAdjusted for age, sex, education level, family history of cancer, past history of chronic disease (dyslipidemia and benign thyroid disease), and total energy intake; ${ }^{b} \mathrm{PTMC}$ with $5 \mathrm{~mm} \leq$ tumor size $<10 \mathrm{~mm}$; ${ }^{\mathrm{c}} \mathrm{Clinical}$ cutpoint and population cutpoint for each thyroid hormone are referenced in the [20], and the [21] mean value based on the data from KNHANE VI (Korea National Health and Nutrition Examination Survey in 2013-2015), respectively; ${ }^{\text {PPop- }}$ ulation cutpoint of $\mathrm{T} 3(\mathrm{ng} / \mathrm{mL})$ derived from controls.

CI, 1.36 to 2.87) (Table 3). The strength of the associations was greater using the clinical cutpoint, than the population cutpoint. In the stratified analyses according to sex, BMI, and women's menopausal status, TSH was positively associated with the PTC risk in females only; and T3 was not associated with PTC or PTMC in males or females (Supplemental Table S2). Graphically, TSH and free T4 showed as U-shaped association with PTC, but the associations were insignificant in those with low levels (Supplemental Fig. S2B, C). In the case of T3 level, it was not significant with PTC in overall level (Supplemental Fig S2D). According to the aggressiveness of PTC in case-only analysis, there was non-significant differences in UICs and thy- roid function were seen between two subgroups (Supplemental Table S3).

The combination of excessive UIC and a high free T4 level was associated with a considerably greater increase in OR of PTC and PTMC than the risk associated with either excessive UIC or a high free T4 level alone (Table 4). The combined effect of excessive UIC and high free T4 levels on risk was more marked for PTC than for PTMC. These suggested that excessive UIC and high free T4 had a synergistic effect. Likewise, the combination of adequate UIC and a TSH level, the risk of PTC was moderately high in the case of the combination with high TSH only. Among individuals with excessive UIC, the risk of 


\begin{tabular}{|c|c|c|c|c|c|c|c|c|}
\hline Variable & & $\begin{array}{l}\text { Controls } \\
(n=463)\end{array}$ & $\begin{array}{c}\text { PTC } \\
(n=209)\end{array}$ & $\begin{array}{c}\text { OR } \\
(95 \% \mathrm{CI})^{\mathrm{a}}\end{array}$ & $\begin{array}{c}\text { OR } \\
(95 \% \mathrm{CI})^{\mathrm{b}}\end{array}$ & $\begin{array}{l}\mathrm{PTMC}^{\mathrm{c}} \\
(n=236)\end{array}$ & $\begin{array}{c}\text { OR } \\
(95 \% \mathrm{CI})^{\mathrm{a}}\end{array}$ & $\begin{array}{c}\text { OR } \\
(95 \% \mathrm{CI})^{\mathrm{b}}\end{array}$ \\
\hline $\mathrm{UIC}, \mu \mathrm{g} / \mathrm{gCr}^{\mathrm{d}}$ & Free $\mathrm{T} 4, \mathrm{ng} / \mathrm{dL}^{\mathrm{d}}$ & & & & & & & \\
\hline \multirow[t]{2}{*}{ Adequate } & Low & 93 & 3 & 1.00 & 1.00 & 6 & 1.00 & 1.00 \\
\hline & High & 106 & 7 & $2.05(0.51-8.14)$ & $2.17(0.52-9.09)$ & 17 & $2.48(0.94-6.57)$ & $3.14(1.07-9.16)$ \\
\hline \multirow[t]{2}{*}{ Excessive } & Low & 160 & 72 & $13.95(4.27-45.53)$ & $18.06(5.23-62.36)$ & 63 & $6.10(2.54-14.65)$ & $7.98(3.00-21.27)$ \\
\hline & High & 104 & 127 & $37.86(11.64-123.0)$ & $43.48(12.63-149.62)$ & 150 & $19.16(8.54-42.98)$ & $26.96(10.26-70.89)$ \\
\hline \multicolumn{9}{|c|}{$\mathrm{UIC}, \mu \mathrm{g} / \mathrm{gCr}^{\mathrm{d}} \quad \mathrm{TSH}, \mu \mathrm{IU} / \mathrm{mL}^{\mathrm{d}}$} \\
\hline \multirow[t]{2}{*}{ Adequate } & Low & 159 & 4 & 1.00 & 1.00 & 13 & 1.00 & 1.00 \\
\hline & High & 40 & 6 & $5.96(1.60-22.13)$ & $4.74(1.21-18.59)$ & 10 & $3.06(0.94-6.57)$ & $2.48(0.91-6.74)$ \\
\hline \multirow[t]{2}{*}{ Excessive } & Low & 164 & 126 & $30.54(11.02-84.61)$ & $35.16(12.15-101.7)$ & 132 & $9.84(5.34-18.12)$ & $10.75(5.39-21.45)$ \\
\hline & High & 100 & 73 & $29.02(10.28-81.86)$ & $32.29(10.88-95.81)$ & 81 & $9.90(5.24-18.73)$ & $10.69(5.16-22.14)$ \\
\hline
\end{tabular}

UIC, urinary iodine concentration; PTC, papillary thyroid cancer; PTMC, papillary thyroid microcarcinoma; OR, odds ratio; CI, confidence interval; T4, thyroxine; TSH, thyroid stimulating hormone.

${ }^{a}$ Crude; ${ }^{b}$ Adjusted for age, sex, education level, family history of cancer, past history of chronic disease (dyslipidemia and benign thyroid disease), and total energy intake; 'PTMC with $5 \mathrm{~mm} \leq$ tumor size $<10 \mathrm{~mm}$; 'UIC levels were divided into two groups: 'Adequate' meant 'UIC $85-219 \mu \mathrm{g} / \mathrm{gCr}$,' and 'excessive' meant 'UIC $\geq 220 \mu \mathrm{g} / \mathrm{gCr}$.' free T4 and TSH levels were divided into two groups using population cutpoints: 'low' and 'high' free T4 levels meant ' $<1.25 \mathrm{ng} / \mathrm{dL}$ ' and ' $\geq 1.25 \mathrm{ng} / \mathrm{dL}$,' respectively; 'low' TSH levels meant ' $<2.16 \mu \mathrm{IU} / \mathrm{mL}$ ' and 'high TSH' $\geq 2.16 \mu \mathrm{IU} / \mathrm{mL}$,' respectively.

PTC and PTMC was high regardless of the TSH levels.

Model performance in each logistic regression model according to PTC and PTMC was higher in the model including the creatinine-adjusted UIC and thyroid function (Model 2, PTC, $\mathrm{AUC}=0.785$; PTMC, $\mathrm{AUC}=0.776)$ than the model including creatinine-adjusted UIC alone (Model 1, PTC, $\mathrm{AUC}=0.714$; PTMC, AUC $=0.688$ ), and the full model showed the highest performance (Model 3, PTC, AUC=0.850; PTMC, AUC $=-$ 0.858 ) (Fig. 1). All of the $P$ values for AUC difference between Model 1 and 2, between Model 2 and 3, and between Model 1 and 3 were statistically significant $(P<0.01)$. Models with creatinine-adjusted UICs were not statistically different from those with unadjusted UICs (Supplemental Table S4).

\section{DISCUSSION}

In this study, individuals with excessive iodine intake had a considerably higher risk of PTC and PTMC than individuals with adequate iodine intake. High free T4 levels were also associated with an increased risk of PTC and PTMC. Excessive UIC had a more marked effect on increasing the risk of PTC than that of PTMC, whereas a high free T4 had a more marked effect on increasing the risk of PTMC than that of PTC. The combined effect of excessive iodine intake and a high free T4 level had a synergistic effect on increasing the risk of PTC and PTMC; whereas in the combination of iodine intake and TSH, a rapid increase in the risk of PTC and PTMC was observed at excessive iodine intake.

Previous results on the association between dietary iodine intake and thyroid cancer have been difficult to interpret because of inconsistencies between studies due to factors such as differences in measurement methods and measurement bias in measuring iodine intake [22,23]. Measurement of 24-hour UIC is the gold standard for assessing iodine intake, but it is not easy to apply at the population level [24]. Therefore, UIC from a random spot urine has been suggested as an alternative indicator $[17,24]$. Even though UIC varies depended on dietary iodine intake, including ionized water drinking, it has been using as a surrogate marker at the population [17,24].

Many previous studies have assessed the association between thyroid cancer and UIC, but some studies have focused on the median difference in UIC between cases and controls $[25,26]$. Although a meta-analysis reported the ORs for PTC relative to normal controls associated with excessive iodine intake [27], we could not directly compare their results with our study for the following reasons: two studies published in Chinese could not identify the method; for another study, the OR reported in the meta-analysis was not reported in the original article [27]; and the fourth study used a UIC reference value that differed from World Health Organization (WHO) standards [27]. In one study the PTC risk associated with an excessive UIC did not differ significantly between cases and controls [28]. In addition, 

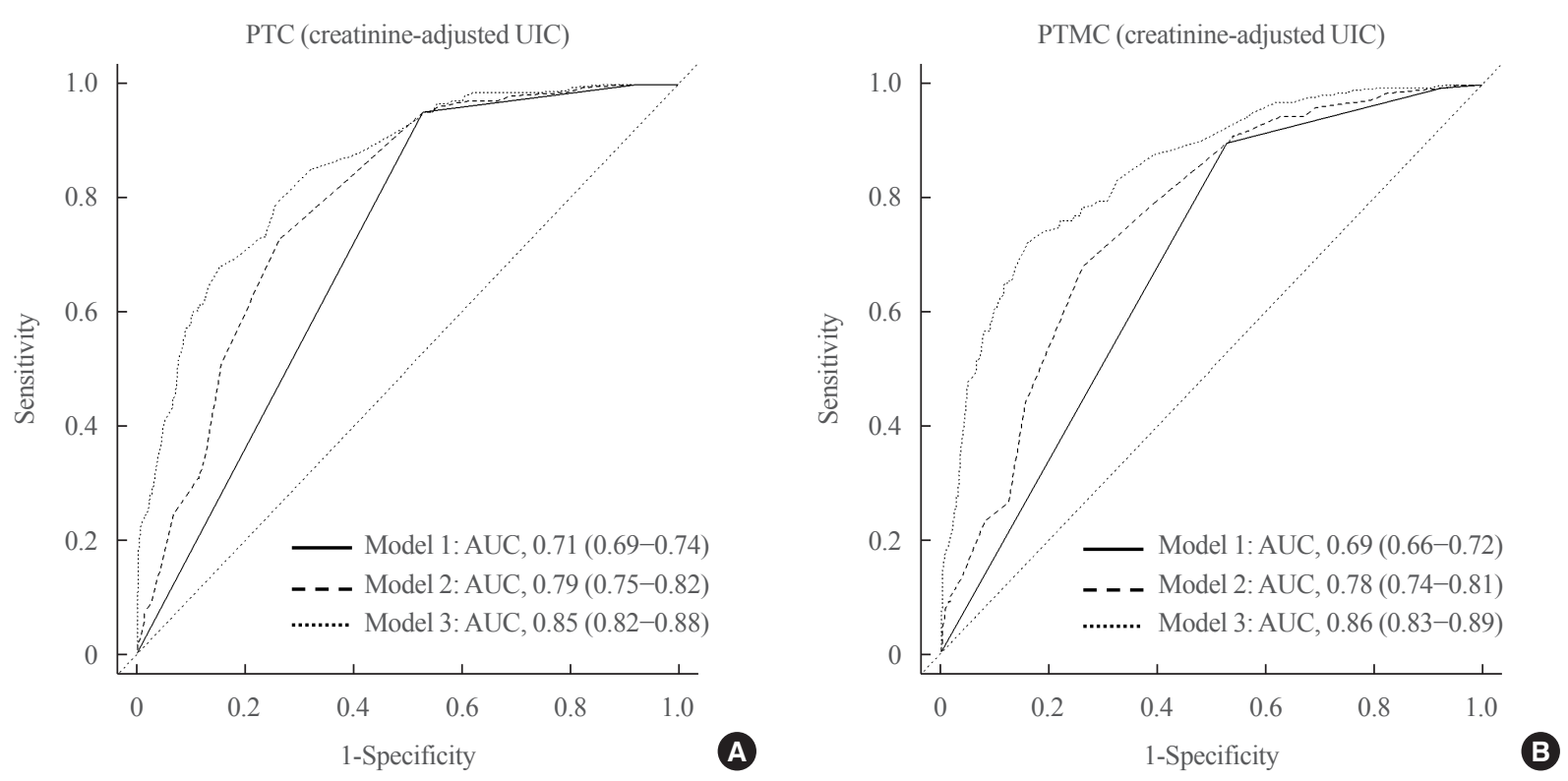

Fig. 1. Area under the curve of receiver operating characteristic (AUC-ROC) between models based on (A) papillary thyroid cancer (PTC) and (B) papillary thyroid microcarcinoma (PTMC) according to creatinine-adjusted urinary iodine concentration (UIC). Model 1 consists of UIC; Model 2 consists of model 1+thyroid stimulating hormone, and free thyroxine level; Model 3 consists of model 2+sex, age, education level, family history of cancer, past history of thyroid disease, dyslipidemia, and total energy intake.

four studies used patients with thyroid nodules as the control group and may have underestimated the effect of UIC on the risk of PTC because iodine and thyroid hormones are involved in thyroid growth and differentiation [28-31].

As in our study, some previous studies have reported the effect of excessive UIC on the clinicopathological aggressiveness of PTC, but their results were inconsistent. Some studies found a relationship between excessive iodine intake and an increased risk of lymph node metastasis [30], larger tumor size [32], capsular invasion [31,32], bilateral location [30], extra-thyroid metastasis [31] and BRAF V600E or T17799A mutation [33,34], while other studies have not found an association between excessive iodine intake and lymph node metastasis [31,32], tumor size $[31,33]$, multifocal tumors or bilaterality [31,32], extracapsular extension [32] or BRAF mutations [26].

In the case of thyroid function and PTC risk, high free T4 level was associated with PTC and PTMC. Similar as the result, previous study reported that high free $\mathrm{T} 4$ level was associated with thyroid cancer. In the case of TSH level, thyroid cancer was associated in moderate TSH level, but it was difficult to identify whether thyroid cancer was pathologically PTC or not [14]. Additionally, several studies considered thyroid peroxidase antibody (TPOAb) and thyroglobulin antibody (TGAb) with TSH and thyroid hormone $[14,28,33,35]$. We identified synergistic effect between excessive iodine intake and high free $\mathrm{T} 4$ levels and an increased risk of PTC and PTMC. However, we could not identify any previous studies that have considered the combined effect of excessive iodine intake and thyroid function on the risk of PTC.

There are possible biological mechanisms that could explain a relationship between excessive iodine intake and high thyroid hormone levels in increasing the risk of PTC. An in vitro study found that thyroid hormone directly contributes to plasma membrane-initiated activation of the mitogen-activated protein kinase (MAPK) cascade, thereby participating in thyroid cancer cell proliferation [36]. In addition, TPOAb and TGAb have been shown to be associated with a significantly increased risk of PTC [35]. This suggested an autoimmune pathogenesis associated with excessive iodine intake [35]; however, the association between the presence of autoantibodies and the risk of PTC had been inconsistent in previous studies [14,29,35]. In the case of TSH, both deficient or excessive iodine intake can increase TSH levels and affect PTC progression [37]. In this process, TSH stimulates thyroid cells, leading to genetic changes and cell proliferation [37]. In the case of low iodine diet, type 1 iodothyronine deiodinase levels increase in the thyroid, leading to TSH stimulation, and an increase in type 2 deiodinase activity in the thyroid [38]. However, excessive iodine intake inhibits pituitary type 2 deiodinase activity, leading to an increase in serum TSH [39]. It has been explained that abnormal iodine in- 
take can lead to thyroid dysfunction by interfering with homeostasis [4-6].

This study has some limitations. First, we did not consider nutritional supplements as additional iodine intake [8]. Second, TPOAb and TGAb were not assessed, so we did not have data on thyroid autoimmunity. Additionally, a single UIC measurement might not consider dietary change. However, a previous study based on KNHANES found that individual dietary changes in iodine intake tend to vary less than that of other nutrients such as high-fat or sugar-based foods. Most foods that serve as iodine sources are served as side dishes rather than as the main dish [40]. Although rare cases were observed in the group with insufficient UIC and low hormone levels (clinical cut), the possibility of bias could be excluded because our results were consistent with those in the ordinal logistic regression analysis, suggested as a correction method for sparse data bias [41]. In addition, since our data was loose matching, we used an unconditional logistic regression model for analysis, which is unlikely to be biased $[42,43]$. Nevertheless, in the analyses for the combined effect of UIC and hormones, since sparse cases were used as a reference group and many covariates were used in the models, there was a possibility of variance inflation. The combined effect of UIC and hormones for the risk of PTC will need to be verified in a larger study in the future.

Despite the limitations, this study also has several strengths. We considered several pathological aggressiveness and the combined effects of thyroid function and iodine intake on PTC and PTMC. Additionally, the overall similarities in the PTC and PTMC results suggests that there is continuity of risk of both PTMC and PTC associated with excessive UIC. Since a lot of eating habits can act as a confounding variable in the association between high iodine intake and thyroid cancer, personal total calorie intake was controlled for all statistical models analyzing the association with iodine intake [12]. We measured UIC by ICP-MS, the gold standard method of UIC, and used creatinine-adjusted UIC as the main explanatory variable to compensate for variations in dietary intake and excretion [44]. Previous studies have shown that creatinine-adjusted UIC is a better indicator the unadjusted UIC because it is less prone to variability and have provided evidence supporting the validity and reliability of creatinine-adjusted UIC $[45,46]$. We also reported the unadjusted UIC results due to their general use in the interpretation of WHO standards.

In the conclusion, this study identified an association between excessive iodine intake and PTC and PTMC. Moreover, we assessed combined effect on PTC and PTMC from iodine intake and thyroid hormones using creatinine-adjusted UIC measured using ICP-MS which is the gold standard measurement method. Considered the effect of iodine intake and thyroid function, this study suggests the it is important to consider the combined effect of iodine intake and thyroid function in order to assess the risk of PTC and PTMC.

\section{CONFLICTS OF INTEREST}

No potential conflict of interest relevant to this article was reported.

\section{ACKNOWLEDGMENTS}

The research was supported by the Korean Foundation for Cancer Research (Grant Number: CB-2017-A-2), the Korea Hydro \& Nuclear Power (KHNP, Grant Number: A15IP12).

\section{AUTHOR CONTRIBUTIONS}

Conception or design: K.K., K.E.L., S.K.P. Acquisition, analysis, or interpretation of data: K.K., S.W.C., Y.J.P., K.E.L., D.W.L., S.K.P. Drafting the work or revising: K.K., S.W.C., Y.J.P., K.E.L. Final approval of the manuscript: K.K., S.W.C., Y.J.P., K.E.L., D.W.L., S.K.P.

\section{ORCID}

Kyungsik Kim https://orcid.org/0000-0001-9007-7025

Sue K. Park https://orcid.org/0000-0001-5002-9707

\section{REFERENCES}

1. International Agency for Research on Cancer, World Health Organization. GLOBOCAN 2020: Global Cancer Observatory, Cancer Today [Internet]. Lyon: International Agency for Research on Cancer; 2021 [cited 2021 Jul 14]. Available from: http://gco.iarc.fr/today.

2. Park S, Oh CM, Cho H, Lee JY, Jung KW, Jun JK, et al. Association between screening and the thyroid cancer "epidemic" in South Korea: evidence from a nationwide study. BMJ 2016;355:i5745.

3. Liu Y, Su L, Xiao H. Review of factors related to the thyroid cancer epidemic. Int J Endocrinol 2017;2017:5308635.

4. Chung HR. Iodine and thyroid function. Ann Pediatr Endocrinol Metab 2014;19:8-12. 
5. Sun X, Shan Z, Teng W. Effects of increased iodine intake on thyroid disorders. Endocrinol Metab (Seoul) 2014;29: 240-7.

6. Zimmermann MB, Boelaert K. Iodine deficiency and thyroid disorders. Lancet Diabetes Endocrinol 2015;3:286-95.

7. Dong W, Zhang H, Zhang P, Li X, He L, Wang Z, et al. The changing incidence of thyroid carcinoma in Shenyang, China before and after universal salt iodization. Med Sci Monit 2013;19:49-53.

8. Blomberg M, Feldt-Rasmussen U, Andersen KK, Kjaer SK. Thyroid cancer in Denmark 1943-2008, before and after iodine supplementation. Int J Cancer 2012;131:2360-6.

9. Bosetti C, Negri E, Kolonel L, Ron E, Franceschi S, Preston-Martin S, et al. A pooled analysis of case-control studies of thyroid cancer. VII. Cruciferous and other vegetables (International). Cancer Causes Control 2002;13:765-75.

10. Cao LZ, Peng XD, Xie JP, Yang FH, Wen HL, Li S. The relationship between iodine intake and the risk of thyroid cancer: a meta-analysis. Medicine (Baltimore) 2017;96:e6734.

11. Zimmermann MB, Galetti V. Iodine intake as a risk factor for thyroid cancer: a comprehensive review of animal and human studies. Thyroid Res 2015;8:8.

12. Arija V, Abellana R, Ribot B, Ramon JM. Biases and adjustments in nutritional assessments from dietary questionnaires. Nutr Hosp 2015;31 Suppl 3:113-8.

13. Teas J, Pino S, Critchley A, Braverman LE. Variability of iodine content in common commercially available edible seaweeds. Thyroid 2004;14:836-41.

14. Cho YA, Kong SY, Shin A, Lee J, Lee EK, Lee YJ, et al. Biomarkers of thyroid function and autoimmunity for predicting high-risk groups of thyroid cancer: a nested casecontrol study. BMC Cancer 2014;14:873.

15. Lee KE, Park YJ, Cho B, Hwang Y, Choi JY, Kim SJ, et al. Protocol of a thyroid cancer longitudinal study (T-CALOS): a prospective, clinical and epidemiological study in Korea. BMJ Open 2015;5:e007234.

16. Ahn J, Lee JH, Lee J, Baek JY, Song E, Oh HS, et al. Association between urinary sodium levels and iodine status in Korea. Korean J Intern Med 2020;35:392-9.

17. WHO/UNICEF/ICCIDD. Assessment of iodine deficiency disorders and monitoring their elimination: a guide for programme managers, 3rd ed [Internet]. Geneva: World Health Organization; 2007 [cited 2021 Jul 14]. Available from: http:// whqlibdoc.who.int/publications/2007/9789241595827_eng. pdf.

18. Munley PH, Bains DS, Bloem WD, Busby RM. Post-trau- matic stress disorder and the MMPI-2. J Trauma Stress 1995;8:171-8.

19. Dasgupta PK, Liu Y, Dyke JV. Iodine nutrition: iodine content of iodized salt in the United States. Environ Sci Technol 2008;42:1315-23.

20. Seoul National University Hospital Clinical Trial Center, SNUH Clinical Lab. Reference Ranges [Internet]. Seoul: Seoul National University Hospital; 2020 [cited 2021 Jul 14]. Available from: http://ctc.bri.snuh.org/researcher/ researcherdown/_notice/7714/download.do?resId=20618.

21. Chung JH. Update on thyroid hormone levels and thyroid dysfunction in the Korean population based on data from the Korea National Health and Nutrition Examination Survey VI (2013 to 2015). Endocrinol Metab (Seoul) 2020;35:7-13.

22. Kipnis V, Midthune D, Freedman L, Bingham S, Day NE, Riboli E, et al. Bias in dietary-report instruments and its implications for nutritional epidemiology. Public Health Nutr 2002;5:915-23.

23. Kipnis V, Subar AF, Midthune D, Freedman LS, BallardBarbash R, Troiano RP, et al. Structure of dietary measurement error: results of the OPEN biomarker study. Am J Epidemiol 2003;158:14-21.

24. Vejbjerg P, Knudsen N, Perrild H, Laurberg P, Andersen S, Rasmussen LB, et al. Estimation of iodine intake from various urinary iodine measurements in population studies. Thyroid 2009;19:1281-6.

25. Hou D, Xu H, Li P, Liu J, Qian Z. Potential role of iodine excess in papillary thyroid cancer and benign thyroid tumor: a case-control study. Asia Pac J Clin Nutr 2020;29:603-8.

26. Lee JH, Song RY, Yi JW, Yu HW, Kwon H, Kim SJ, et al. Case-control study of papillary thyroid carcinoma on urinary and dietary iodine status in South Korea. World J Surg 2018;42:1424-31.

27. Yan AR, Zhang X, Shen H, Zhou X, Li R, Yuan Z. Urinary iodine is increased in papillary thyroid carcinoma but is not altered by regional population iodine intake status: a metaanalysis and implications. Endocr J 2019;66:497-514.

28. Zhao H, Li H, Huang T. High urinary iodine, thyroid autoantibodies, and thyroid-stimulating hormone for papillary thyroid cancer risk. Biol Trace Elem Res 2018;184:317-24.

29. Kim HJ, Kim NK, Park HK, Byun DW, Suh K, Yoo MH, et al. Strong association of relatively low and extremely excessive iodine intakes with thyroid cancer in an iodine-replete area. Eur J Nutr 2017;56:965-71.

30. Xiu C, He Q, Zhao HJ, Yuan ZN, Guo LH, Wang FQ, et al. Strong correlation of abnormal serum and urinary iodine

Copyright (C) 2021 Korean Endocrine Society 
levels with papillary thyroid cancer: a case-control study. Biomed Environ Sci 2020;33:62-7.

31. Huang F, Cong W, Xiao J, Zhou Y, Gong M, Sun J, et al. Association between excessive chronic iodine exposure and the occurrence of papillary thyroid carcinoma. Oncol Lett 2020;20:189.

32. Zhao H, Li H, Huang T. High iodine intake and central lymph node metastasis risk of papillary thyroid cancer. J Trace Elem Med Biol 2019;53:16-21.

33. Kim HJ, Park HK, Byun DW, Suh K, Yoo MH, Min YK, et al. Iodine intake as a risk factor for BRAF mutations in papillary thyroid cancer patients from an iodine-replete area. Eur J Nutr 2018;57:809-15.

34. Guan H, Ji M, Bao R, Yu H, Wang Y, Hou P, et al. Association of high iodine intake with the T1799A BRAF mutation in papillary thyroid cancer. J Clin Endocrinol Metab 2009; 94:1612-7.

35. Wu X, Lun Y, Jiang H, Gang Q, Xin S, Duan Z, et al. Coexistence of thyroglobulin antibodies and thyroid peroxidase antibodies correlates with elevated thyroid-stimulating hormone level and advanced tumor stage of papillary thyroid cancer. Endocrine 2014;46:554-60.

36. Lin HY, Tang HY, Shih A, Keating T, Cao G, Davis PJ, et al. Thyroid hormone is a MAPK-dependent growth factor for thyroid cancer cells and is anti-apoptotic. Steroids 2007;72: 180-7.

37. McLeod DS. Thyrotropin in the development and management of differentiated thyroid cancer. Endocrinol Metab Clin North Am 2014;43:367-83.

38. Lavado-Autric R, Calvo RM, de Mena RM, de Escobar GM, Obregon MJ. Deiodinase activities in thyroids and tissues of iodine-deficient female rats. Endocrinology 2013; 154:529-36.

39. Li N, Jiang Y, Shan Z, Teng W. Prolonged high iodine intake is associated with inhibition of type 2 deiodinase activity in pituitary and elevation of serum thyrotropin levels. Br J Nutr 2012;107:674-82.

40. Ko YM, Kwon YS, Park YK. An iodine database establishment and iodine intake in Korean adults: based on the 1998 2014 Korea National Health and Nutrition Examination Survey. J Nutr Health 2017;50:624-44.

41. Lipsitz SR, Fitzmaurice GM, Regenbogen SE, Sinha D, Ibrahim JG, Gawande AA. Bias correction for the proportional odds logistic regression model with application to a study of surgical complications. J R Stat Soc Ser C Appl Stat 2013;62:233-50.

42. Walker DA, Smith TJ. Logistic regression under sparse data conditions. J Mod Appl Stat Methods 2019;18:eP3372.

43. Kuo CL, Duan Y, Grady J. Unconditional or conditional logistic regression model for age-matched case-control data? Front Public Health 2018;6:57.

44. Caldwell KL, Maxwell CB, Makhmudov A, Pino S, Braverman LE, Jones RL, et al. Use of inductively coupled plasma mass spectrometry to measure urinary iodine in NHANES 2000: comparison with previous method. Clin Chem 2003;49 (6 Pt 1):1019-21.

45. Knudsen N, Christiansen E, Brandt-Christensen M, Nygaard B, Perrild H. Age- and sex-adjusted iodine/creatinine ratio. A new standard in epidemiological surveys? Evaluation of three different estimates of iodine excretion based on casual urine samples and comparison to $24 \mathrm{~h}$ values. Eur J Clin Nutr 2000;54:361-3.

46. Kim HK, Lee SY, Lee JI, Jang HW, Kim SK, Chung HS, et al. Usefulness of iodine/creatinine ratio from spot-urine samples to evaluate the effectiveness of low-iodine diet preparation for radioiodine therapy. Clin Endocrinol (Oxf) 2010;73: 114-8. 